\title{
Ostrowski Via a Two Functions Pompeiu's Inequality
}

\section{S. S. Dragomir}

\begin{abstract}
In this paper, some generalizations of Pompeiu's inequality for two complex-valued absolutely continuous functions are provided. They are applied to obtain some new Ostrowski type results. Reverses for the integral Cauchy-Bunyakovsky-Schwarz inequality are provided as well.
\end{abstract}

\section{Introduction}

In 1946, Pompeiu [6] derived a variant of Lagrange's mean value theorem, now known as Pompeiu's mean value theorem (see also [8, p. 83]).

Theorem 1 (Pompeiu, 1946 [6]). For every real valued function $f$ differentiable on an interval $[a, b]$ not containing 0 and for all pairs $x_{1} \neq x_{2}$ in $[a, b]$, there exists a point $\xi$ between $x_{1}$ and $x_{2}$ such that

$$
\frac{x_{1} f\left(x_{2}\right)-x_{2} f\left(x_{1}\right)}{x_{1}-x_{2}}=f(\xi)-\xi f^{\prime}(\xi) .
$$

The following inequality is useful to derive some Ostrowski type inequalities.

Key Words: Ostrowski's inequality, Pompeiu's mean value theorem, Integral inequalities, Schwarz inequality

2010 Mathematics Subject Classification: Primary 26D15; Secondary 26D10

Received: 23.03.2015

Revised: 10.07 .2015

Accepted: 21.07.2015 
Corollary 1 (Pompeiu's Inequality). With the assumptions of Theorem 1 and if $\left\|f-\ell f^{\prime}\right\|_{\infty}=\sup _{t \in(a, b)}\left|f(t)-t f^{\prime}(t)\right|<\infty$ where $\ell(t)=t, t \in[a, b]$, then

$$
|t f(x)-x f(t)| \leq\left\|f-\ell f^{\prime}\right\|_{\infty}|x-t|
$$

for any $t, x \in[a, b]$.

The inequality (2) was stated by the author in [3].

In 1938, A. Ostrowski [4] proved the following result in the estimating the integral mean:

Theorem 2 (Ostrowski, $1938[4]$ ). Let $f:[a, b] \rightarrow \mathbb{R}$ be continuous on $[a, b]$ and differentiable on $(a, b)$ with $\left|f^{\prime}(t)\right| \leq M<\infty$ for all $t \in(a, b)$. Then for any $x \in[a, b]$, we have the inequality

$$
\left|f(x)-\frac{1}{b-a} \int_{a}^{b} f(t) d t\right| \leq\left[\frac{1}{4}+\left(\frac{x-\frac{a+b}{2}}{b-a}\right)^{2}\right] M(b-a) .
$$

The constant $\frac{1}{4}$ is best possible in the sense that it cannot be replaced by a smaller quantity.

In order to provide another approximation of the integral mean, by making use of the Pompeiu's mean value theorem, the author proved the following result:

Theorem 3 (Dragomir, $2005[3]$ ). Let $f:[a, b] \rightarrow \mathbb{R}$ be continuous on $[a, b]$ and differentiable on $(a, b)$ with $[a, b]$ not containing 0 . Then for any $x \in[a, b]$, we have the inequality

$$
\begin{aligned}
& \left|\frac{a+b}{2} \cdot \frac{f(x)}{x}-\frac{1}{b-a} \int_{a}^{b} f(t) d t\right| \\
& \leq \frac{b-a}{|x|}\left[\frac{1}{4}+\left(\frac{x-\frac{a+b}{2}}{b-a}\right)^{2}\right]\left\|f-\ell f^{\prime}\right\|_{\infty},
\end{aligned}
$$

where $\ell(t)=t, t \in[a, b]$.

The constant $\frac{1}{4}$ is sharp in the sense that it cannot be replaced by a smaller quantity.

In [7], E. C. Popa using a mean value theorem obtained a generalization of (4) as follows: 
Theorem 4 (Popa, $2007[7])$. Let $f:[a, b] \rightarrow \mathbb{R}$ be continuous on $[a, b]$ and differentiable on $(a, b)$. Assume that $\alpha \notin[a, b]$. Then for any $x \in[a, b]$, we have the inequality

$$
\begin{aligned}
& \left|\left(\frac{a+b}{2}-\alpha\right) f(x)+\frac{\alpha-x}{b-a} \int_{a}^{b} f(t) d t\right| \\
& \leq\left[\frac{1}{4}+\left(\frac{x-\frac{a+b}{2}}{b-a}\right)^{2}\right](b-a)\left\|f-\ell_{\alpha} f^{\prime}\right\|_{\infty},
\end{aligned}
$$

where $\ell_{\alpha}(t)=t-\alpha, t \in[a, b]$.

In [5], J. Pečarić and S. Ungar have proved a general estimate with the $p$-norm, $1 \leq p \leq \infty$ which for $p=\infty$ gives Dragomir's result.

Theorem 5 (Pečarić \& Ungar, $2006[5])$. Let $f:[a, b] \rightarrow \mathbb{R}$ be continuous on $[a, b]$ and differentiable on $(a, b)$ with $0<a<b$. Then for $1 \leq p, q \leq \infty$ with $\frac{1}{p}+\frac{1}{q}=1$ we have the inequality

$$
\left|\frac{a+b}{2} \cdot \frac{f(x)}{x}-\frac{1}{b-a} \int_{a}^{b} f(t) d t\right| \leq P U(x, p)\left\|f-\ell f^{\prime}\right\|_{p},
$$

for $x \in[a, b]$, where

$$
\begin{aligned}
P U(x, p): & =(b-a)^{\frac{1}{p}-1}\left[\left(\frac{a^{2-q}-x^{2-q}}{(1-2 q)(2-q)}+\frac{x^{2-q}-a^{1+q} x^{1-2 q}}{(1-2 q)(1+q)}\right)^{1 / q}\right. \\
& \left.+\left(\frac{b^{2-q}-x^{2-q}}{(1-2 q)(2-q)}+\frac{x^{2-q}-b^{1+q} x^{1-2 q}}{(1-2 q)(1+q)}\right)^{1 / q}\right] .
\end{aligned}
$$

In the cases $(p, q)=(1, \infty),(\infty, 1)$ and $(2,2)$ the quantity $P U(x, p)$ has to be taken as the limit as $p \rightarrow 1, \infty$ and 2 , respectively.

For other inequalities in terms of the $p$-norm of the quantity $f-\ell_{\alpha} f^{\prime}$, where $\ell_{\alpha}(t)=t-\alpha, t \in[a, b]$ and $\alpha \notin[a, b]$ see [1] and [2].

In this paper, some new Pompeiu's type inequalities for two complex-valued absolutely continuous functions are provided. They are applied to obtain some Ostrowski type inequalities. Reverses for the integral Cauchy-BunyakovskySchwarz inequality are provided as well.

\section{A General Pompeiu's Inequality}

We start with the following generalization of (2). 
Theorem 6. Let $f, g:[a, b] \rightarrow \mathbb{C}$ be absolutely continuous functions on the interval $[a, b]$ with $g(t) \neq 0$ for all $t \in[a, b]$. Then for any $t, x \in[a, b]$ we have

$$
\begin{aligned}
& \left|\frac{f(x)}{g(x)}-\frac{f(t)}{g(t)}\right| \\
& \leq\left\{\begin{array}{lc}
\left\|f^{\prime} g-f g^{\prime}\right\|_{\infty}\left|\int_{t}^{x} \frac{1}{|g(s)|^{2}} d s\right| & \text { if } f^{\prime} g-f g^{\prime} \in L_{\infty}[a, b], \\
\left\|f^{\prime} g-f g^{\prime}\right\|_{p}\left|\int_{t}^{x} \frac{1}{|g(s)|^{2 q}} d s\right|^{1 / q} & \text { if } f^{\prime} g-f g^{\prime} \in L_{p}[a, b] \\
p>1, \\
\frac{1}{p}+\frac{1}{q}=1,
\end{array}\right. \\
& \left\|f^{\prime} g-f g^{\prime}\right\|_{1} \sup _{s \in[t, x]([x, t])}\left\{\frac{1}{|g(s)|^{2}}\right\}
\end{aligned}
$$

or, equivalently

$$
\begin{aligned}
& |g(t) f(x)-f(t) g(x)| \\
\leq & \left\{\begin{array}{lc}
\left\|f^{\prime} g-f g^{\prime}\right\|_{\infty}|g(t) g(x)|\left|\int_{t}^{x} \frac{1}{|g(s)|^{2}} d s\right| & \text { if } f^{\prime} g-f g^{\prime} \in L_{\infty}[a, b], \\
\left\|f^{\prime} g-f g^{\prime}\right\|_{p}|g(t) g(x)|\left|\int_{t}^{x} \frac{1}{|g(s)|^{2 q}} d s\right| \begin{array}{cc}
1 / q & \text { if } f^{\prime} g-f g^{\prime} \in L_{p}[a, b] \\
p>1, \\
\frac{1}{p}+\frac{1}{q}=1,
\end{array} \\
\left\|f^{\prime} g-f g^{\prime}\right\|_{1}|g(t) g(x)| \sup _{s \in[t, x]([x, t])}\left\{\frac{1}{|g(s)|^{2}}\right\} .
\end{array}\right.
\end{aligned}
$$

Proof. If $f$ and $g$ are absolutely continuous and $g(t) \neq 0$ for all $t \in[a, b]$, then $f / g$ is absolutely continuous on the interval $[a, b]$ and

$$
\int_{t}^{x}\left(\frac{f(s)}{g(s)}\right)^{\prime} d s=\frac{f(x)}{g(x)}-\frac{f(t)}{g(t)}
$$

for any $t, x \in[a, b]$ with $x \neq t$.

Since

$$
\int_{t}^{x}\left(\frac{f(s)}{g(s)}\right)^{\prime} d s=\int_{t}^{x} \frac{f^{\prime}(s) g(s)-f(s) g^{\prime}(s)}{g^{2}(s)} d s
$$

then we get the following identity

$$
\frac{f(x)}{g(x)}-\frac{f(t)}{g(t)}=\int_{t}^{x} \frac{f^{\prime}(s) g(s)-f(s) g^{\prime}(s)}{g^{2}(s)} d s
$$


for any $t, x \in[a, b]$.

Taking the modulus in (9) we have

$$
\begin{aligned}
\left|\frac{f(x)}{g(x)}-\frac{f(t)}{g(t)}\right| & =\left|\int_{t}^{x} \frac{f^{\prime}(s) g(s)-f(s) g^{\prime}(s)}{g^{2}(s)} d s\right| \\
& \leq\left|\int_{t}^{x} \frac{\left|f^{\prime}(s) g(s)-f(s) g^{\prime}(s)\right|}{|g(s)|^{2}} d s\right|:=I
\end{aligned}
$$

and utilizing Hölder's integral inequality we deduce

$$
\begin{aligned}
I \leq\left\{\begin{array}{cc}
\sup _{s \in[t, x]([x, t])}\left|f^{\prime}(s) g(s)-f(s) g^{\prime}(s)\right|\left|\int_{t}^{x} \frac{1}{|g(s)|^{2}} d s\right|, \\
\left|\int_{t}^{x}\right| f^{\prime}(s) g(s)-\left.\left.f(s) g^{\prime}(s)\right|^{p} d s\right|^{1 / p}\left|\int_{t}^{x} \frac{1}{|g(s)|^{2 q}} d s\right|^{1 / q} & \begin{array}{c}
p>1, \\
p
\end{array}+\frac{1}{q}=1, \\
\left|\int_{t}^{x}\right| f^{\prime}(s) g(s)-f(s) g^{\prime}(s)|d s| \sup _{s \in[t, x]([x, t])}\left\{\frac{1}{|g(s)|^{2}}\right\},
\end{array}\right. \\
\leq\left\{\begin{array}{cc}
\left\|f^{\prime} g-f g^{\prime}\right\|_{\infty}\left|\int_{t}^{x} \frac{1}{|g(s)|^{2}} d s\right|, & p>1, \\
\left\|f^{\prime} g-f g^{\prime}\right\|_{p}\left|\int_{t}^{x} \frac{1}{|g(s)|^{2 q}} d s\right|^{1 / q} & \frac{1}{p}+\frac{1}{q}=1, \\
\left\|f^{\prime} g-f g^{\prime}\right\|_{1} \sup _{s \in[t, x]([x, t])}\left\{\frac{1}{|g(s)|^{2}}\right\} &
\end{array}\right.
\end{aligned}
$$

and the inequality (7) is proved.

The following particular case extends Pompeiu's inequality to other $p$ norms than $p=\infty$ obtained in (2).

Corollary 2. Let $f:[a, b] \rightarrow \mathbb{C}$ be an absolutely continuous function on the interval $[a, b]$ with $b>a>0$. Then for any $t, x \in[a, b]$ we have 


$$
\begin{aligned}
& \left|\frac{f(x)}{x}-\frac{f(t)}{t}\right| \\
& \leq\left\{\begin{array}{lc}
\left\|f-\ell f^{\prime}\right\|_{\infty}\left|\frac{1}{t}-\frac{1}{x}\right| & \text { if } f-\ell f^{\prime} \in L_{\infty}[a, b], \\
\frac{1}{(2 q-1)^{1 / q}}\left\|f-\ell f^{\prime}\right\|_{p}\left|\frac{1}{t^{2 q-1}}-\frac{1}{x^{2 q-1}}\right|^{1 / q} & \text { if } f-\ell f^{\prime} \in L_{p}[a, b] \\
\left\|f-\ell f^{\prime}\right\|_{1} \frac{1}{\min \left\{t^{2}, x^{2}\right\}} & \frac{1}{p}+\frac{1}{q}=1,
\end{array}\right.
\end{aligned}
$$

or, equivalently

$$
\begin{aligned}
& |t f(x)-x f(t)| \\
& \quad \leq \begin{cases}\left\|f-\ell f^{\prime}\right\|_{\infty}|x-t| & \text { if } f-\ell f^{\prime} \in L_{\infty}[a, b], \\
\frac{1}{(2 q-1)^{1 / q}}\left\|f-\ell f^{\prime}\right\|_{p}\left|\frac{x^{q}}{t^{q-1}}-\frac{t^{q}}{x^{q-1}}\right|^{1 / q} & \text { if } f-\ell f^{\prime} \in L_{p}[a, b] \\
\left\|f-\ell f^{\prime}\right\|_{1} \frac{\max \{t, x\}}{\min \{t, x\}}, & \end{cases}
\end{aligned}
$$

where $\ell(t)=t, t \in[a, b]$.

The proof follows by (7) for $g(t)=\ell(t)=t, t \in[a, b]$.

The general case for power functions is as follows.

Corollary 3. Let $f:[a, b] \rightarrow \mathbb{C}$ be an absolutely continuous function on the interval $[a, b]$ with $b>a>0$. If $r \in \mathbb{R}, r \neq 0$, then for any $t, x \in[a, b]$ we have 


$$
\begin{aligned}
& \left|\frac{f(x)}{x^{r}}-\frac{f(t)}{t^{r}}\right| \\
& \leq\left\{\begin{array}{l}
\frac{1}{|r|}\left\|f^{\prime} \ell-r f\right\|_{\infty}\left|\frac{1}{x^{r}}-\frac{1}{t^{r}}\right|, \text { if } f^{\prime} \ell-r f \in L_{\infty}[a, b], \\
\left\|f^{\prime} \ell-r f\right\|_{p} \\
\times\left\{\begin{array}{l}
\quad \frac{1}{|1-q(r+1)|^{1 / q}}\left|\frac{1}{x^{1-q(r+1)}}-\frac{1}{t^{1-q(r+1)}}\right|^{1 / q}, \text { for } r \neq-\frac{1}{p} \\
|\ln x-\ln t|^{1 / q}, \text { for } r=-\frac{1}{p}
\end{array}\right. \\
\text { if } f^{\prime} \ell-r f \in L_{p}[a, b], \\
\left\|f^{\prime} \ell-r f\right\|_{1} \frac{1}{\min \left\{x^{r+1}, t^{r+1}\right\}},
\end{array}\right.
\end{aligned}
$$

or, equivalently

$$
\begin{aligned}
& \left|t^{r} f(x)-x^{r} f(t)\right| \\
& \leq\left\{\begin{array}{l}
\frac{1}{|r|}\left\|f^{\prime} \ell-r f\right\|_{\infty}\left|t^{r}-x^{r}\right|, \text { if } f^{\prime} \ell-r f \in L_{\infty}[a, b], \\
\left\|f^{\prime} \ell-r f\right\|_{p} \\
\times\left\{\begin{array}{l}
\frac{t^{r} x^{r}}{|1-q(r+1)|^{1 / q}}\left|\frac{1}{x^{1-q(r+1)}}-\frac{1}{t^{1-q(r+1)}}\right|^{1 / q}, \text { for } r \neq-\frac{1}{p} \\
t^{r} x^{r}|\ln x-\ln t|^{1 / q}, \text { for } r=-\frac{1}{p}
\end{array}\right. \\
\text { if } f^{\prime} \ell-r f \in L_{p}[a, b], \\
\left\|f^{\prime} \ell-r f\right\|_{1} \frac{t^{r} x^{r}}{\min \left\{x^{r+1}, t^{r+1}\right\}},
\end{array}\right.
\end{aligned}
$$

where $p>1, \frac{1}{p}+\frac{1}{q}=1$.

The proof follows by $(7)$ for $g(t)=t^{r}, t \in[a, b]$. The details for calculations are omitted.

We have the following result for exponential.

Corollary 4. Let $f:[a, b] \rightarrow \mathbb{C}$ be an absolutely continuous function on the 
interval $[a, b]$ and $\alpha \in \mathbb{R}, \alpha \neq 0$. Then for any $t, x \in[a, b]$ we have

$$
\begin{aligned}
\left|\frac{f(x)}{\exp (i \alpha x)}-\frac{f(t)}{\exp (i \alpha t)}\right| & \begin{cases}\left\|f^{\prime}-i \alpha f\right\|_{\infty}|x-t| & \text { if } f^{\prime}-i \alpha f \in L_{\infty}[a, b], \\
\left\|f^{\prime}-i \alpha f\right\|_{p}|x-t|^{1 / q} & \text { if } f^{\prime}-i \alpha f \in L_{p}[a, b] \\
& p>1, \\
& \frac{1}{p}+\frac{1}{q}=1, \\
\left\|f^{\prime}-i \alpha f\right\|_{1} & \end{cases}
\end{aligned}
$$

or, equivalently

$$
\begin{aligned}
& |\exp (i \alpha t) f(x)-f(t) \exp (i \alpha x)| \\
& \leq \begin{cases}\left\|f^{\prime}-i \alpha f\right\|_{\infty}|x-t| & \text { if } f^{\prime}-i \alpha f \in L_{\infty}[a, b], \\
\left\|f^{\prime}-i \alpha f\right\|_{p}|x-t|^{1 / q} & \text { if } f^{\prime}-i \alpha f \in L_{p}[a, b] \\
& \frac{1}{p}+\frac{1}{q}=1, \\
\left\|f^{\prime}-i \alpha f\right\|_{1} .\end{cases}
\end{aligned}
$$

\section{An Inequality Generalizing Ostrowski's}

The following result holds:

Theorem 7. Let $f, g:[a, b] \rightarrow \mathbb{C}$ be absolutely continuous functions on the interval $[a, b]$. If $0<m \leq|g(t)| \leq M<\infty$ for any $t \in[a, b]$, then 


$$
\begin{aligned}
& \left|f(x) \int_{a}^{b} g(t) d t-g(x) \int_{a}^{b} f(t) d t\right| \\
& \leq\left(\frac{M}{m}\right)^{2}\left\{\begin{array}{cc}
\left\|f^{\prime} g-f g^{\prime}\right\|_{\infty}(b-a)^{2}\left[\frac{1}{4}+\left(\frac{x-\frac{a+b}{2}}{b-a}\right)^{2}\right] & \text { if } f^{\prime} g-f g^{\prime} \in L_{\infty}[a, b], \\
\left\|f^{\prime} g-f g^{\prime}\right\|_{p}\left[\frac{(b-x)^{1+1 / q}+(x-a)^{1+1 / q}}{1+1 / q}\right] & \text { if } f^{\prime} g-f g^{\prime} \in L_{p}[a, b] \\
p>1, \\
\frac{1}{p}+\frac{1}{q}=1, \\
\left\|f^{\prime} g-f g^{\prime}\right\|_{1}(b-a)
\end{array}\right.
\end{aligned}
$$

for any $x \in[a, b]$.

Proof. Utilizing (8) we have

$$
\begin{gathered}
\left|f(x) \int_{a}^{b} g(t) d t-g(x) \int_{a}^{b} f(t) d t\right| \\
\leq \int_{a}^{b}|g(t) f(x)-f(t) g(x)| d t \\
\leq\left\{\begin{array}{l}
\left\|f^{\prime} g-f g^{\prime}\right\|_{\infty}|g(x)| \int_{a}^{b}\left(|g(t)|\left|\int_{t}^{x} \frac{1}{|g(s)|^{2}} d s\right|\right) d t, \\
\left\|f^{\prime} g-f g^{\prime}\right\|_{p}|g(x)| \int_{a}^{b}\left(|g(t)|\left|\int_{t}^{x} \frac{1}{|g(s)|^{2 q}} d s\right|^{1 / q}\right) d t, \\
\left\|f^{\prime} g-f g^{\prime}\right\|_{1}|g(x)| \int_{a}^{b}\left(|g(t)| \sup _{s \in[t, x]([x, t])}\left\{\frac{1}{|g(s)|^{2}}\right\}\right) d t
\end{array}\right.
\end{gathered}
$$

for any $x \in[a, b]$, which is of interest in itself. 
Since $0<m \leq|g(t)| \leq M<\infty$ for any $t \in[a, b]$, then

$$
\begin{array}{r}
|g(x)| \int_{a}^{b}\left(|g(t)|\left|\int_{t}^{x} \frac{1}{|g(s)|^{2}} d s\right|\right) d t \leq\left(\frac{M}{m}\right)^{2} \int_{a}^{b}|x-t| d t \\
=\left(\frac{M}{m}\right)^{2}\left[\frac{1}{4}+\left(\frac{x-\frac{a+b}{2}}{b-a}\right)^{2}\right] \\
|g(x)| \int_{a}^{b}\left(|g(t)|\left|\int_{t}^{x} \frac{1}{|g(s)|^{2 q}} d s\right|^{1 / q}\right) d t \\
\leq\left(\frac{M}{m}\right)^{2} \int_{a}^{b}|x-t|^{1 / q} d t=\left(\frac{M}{m}\right)^{2} \frac{(b-x)^{1+1 / q}+(x-a)^{1+1 / q}}{1+1 / q}
\end{array}
$$

and

$|g(x)| \int_{a}^{b}\left(|g(t)| \sup _{s \in[t, x]([x, t])}\left\{\frac{1}{|g(s)|^{2}}\right\}\right) d t \leq\left(\frac{M}{m}\right)^{2} \int_{a}^{b} d t=\left(\frac{M}{m}\right)^{2}(b-a)$

for any $x \in[a, b]$ and by (18) we get the desired result (17).

Remark 1. If we take $g(t)=1, t \in[a, b]$ in the first inequality (17) we recapture Ostrowski's inequality.

Corollary 5. With the assumptions in Theorem 7 we have the midpoint inequalities

$$
\begin{aligned}
& \left|f\left(\frac{a+b}{2}\right) \int_{a}^{b} g(t) d t-g\left(\frac{a+b}{2}\right) \int_{a}^{b} f(t) d t\right| \\
& \leq\left(\frac{M}{m}\right)^{2}\left\{\begin{array}{cc}
\frac{1}{4}(b-a)^{2}\left\|f^{\prime} g-f g^{\prime}\right\|_{\infty} & \text { if } f^{\prime} g-f g^{\prime} \in L_{\infty}[a, b], \\
\frac{1}{2^{1 / q}(1+1 / q)}(b-a)^{1+1 / q}\left\|f^{\prime} g-f g^{\prime}\right\|_{p} \begin{array}{c}
\text { if } f^{\prime} g-f g^{\prime} \in L_{p}[a, b] \\
\frac{1}{p}+\frac{1}{q}=1 .
\end{array}
\end{array}\right.
\end{aligned}
$$

The following result also holds: 
Theorem 8. Let $f, g:[a, b] \rightarrow \mathbb{C}$ be absolutely continuous functions on the interval $[a, b], g(x) \neq 0$ for $x \in[a, b]$ and $g^{-2} \in L_{\infty}[a, b]$. Then

$$
\begin{aligned}
& \left|\frac{f(x)}{g(x)} \int_{a}^{b} g(t) d t-\int_{a}^{b} f(t) d t\right| \\
& \leq\left\|g^{-2}\right\|_{\infty} \times \begin{cases}\left\|f^{\prime} g-f g^{\prime}\right\|_{\infty} \int_{a}^{b}|g(t)||x-t| d t, & \text { if } f^{\prime} g-f g^{\prime} \in L_{\infty}[a, b], \\
\left\|f^{\prime} g-f g^{\prime}\right\|_{p} \int_{a}^{b}|g(t)||x-t|^{1 / q} d t & \text { if } f^{\prime} g-f g^{\prime} \in L_{p}[a, b] \\
p>1, & \frac{1}{p}+\frac{1}{q}=1, \\
\left\|f^{\prime} g-f g^{\prime}\right\|_{1} \int_{a}^{b}|g(t)| d t & \end{cases}
\end{aligned}
$$

for any $x \in[a, b]$.

Proof. Utilizing (8) we have

$$
\begin{aligned}
& \left|\frac{f(x)}{g(x)} \int_{a}^{b} g(t) d t-\int_{a}^{b} f(t) d t\right| \\
& \leq\left\{\begin{array}{l}
\left\|f^{\prime} g-f g^{\prime}\right\|_{\infty} \int_{a}^{b}\left(|g(t)|\left|\int_{t}^{x} \frac{1}{|g(s)|^{2}} d s\right|\right) d t \\
\left\|f^{\prime} g-f g^{\prime}\right\|_{p} \int_{a}^{b}\left(|g(t)|\left|\int_{t}^{x} \frac{1}{|g(s)|^{2 q}} d s\right|^{1 / q}\right) d t \\
\left\|f^{\prime} g-f g^{\prime}\right\|_{1} \int_{a}^{b}\left(|g(t)| \sup _{s \in[t, x]([x, t])}\left\{\frac{1}{|g(s)|^{2}}\right\}\right) d t
\end{array}\right.
\end{aligned}
$$

for any $x \in[a, b]$.

Since

$$
\begin{gathered}
\left|\int_{t}^{x} \frac{1}{|g(s)|^{2}} d s\right| \leq\left\|g^{-2}\right\|_{\infty}|x-t|, \\
\left|\int_{t}^{x} \frac{1}{|g(s)|^{2 q}} d s\right|^{1 / q} \leq\left\|g^{-2}\right\|_{\infty}|x-t|^{1 / q}
\end{gathered}
$$

and

$$
\sup _{s \in[t, x]([x, t])}\left\{\frac{1}{|g(s)|^{2}}\right\} \leq\left\|g^{-2}\right\|_{\infty}
$$


for any $x, t \in[a, b]$, then on making use of (21) we get the desired result (20).

We have the midpoint inequalities:

Corollary 6. With the assumptions of Theorem 8 we have

$$
\begin{aligned}
& \left|\frac{f\left(\frac{a+b}{2}\right)}{g\left(\frac{a+b}{2}\right)} \int_{a}^{b} g(t) d t-\int_{a}^{b} f(t) d t\right| \\
& \leq\left\|g^{-2}\right\|_{\infty} \times\left\{\begin{array}{lc}
\left\|f^{\prime} g-f g^{\prime}\right\|_{\infty} \int_{a}^{b}|g(t)|\left|\frac{a+b}{2}-t\right| d t, & \text { if } f^{\prime} g-f g^{\prime} \in L_{\infty}[a, b], \\
\left\|f^{\prime} g-f g^{\prime}\right\|_{p} \int_{a}^{b}|g(t)|\left|\frac{a+b}{2}-t\right|^{1 / q} d t & \text { if } f^{\prime} g-f g^{\prime} \in L_{p}[a, b] \\
p>1, \\
\frac{1}{p}+\frac{1}{q}=1 .
\end{array}\right.
\end{aligned}
$$

We have the following exponential version of Ostrowski's inequality as well:

Theorem 9. Let $f:[a, b] \rightarrow \mathbb{C}$ be an absolutely continuous function on the interval $[a, b]$ and $\alpha \in \mathbb{R}, \alpha \neq 0$. Then for any $x \in[a, b]$ we have

$$
\begin{aligned}
& \left|\frac{\exp (i \alpha(b-x))-\exp (-i \alpha(x-a))}{i \alpha} f(x)-\int_{a}^{b} f(t) d t\right| \\
& \leq \begin{cases}\left\|f^{\prime}-i \alpha f\right\|_{\infty}(b-a)^{2}\left[\frac{1}{4}+\left(\frac{t-\frac{a+b}{2}}{b-a}\right)^{2}\right], & \text { if } f^{\prime}-i \alpha f \in L_{\infty}[a, b] \\
\left\|f^{\prime}-i \alpha f\right\|_{p} \frac{(b-x)^{1+1 / q}+(x-a)^{1+1 / q}}{1+1 / q}, & \in L_{p}[a, b] \\
& p>1, \\
& \frac{1}{p}+\frac{1}{q}=1 \\
\left\|f^{\prime}-i \alpha f\right\|_{1} .\end{cases}
\end{aligned}
$$


Proof. If we write the inequality (18) for $g(t)=\exp (i \alpha t), t \in[a, b]$, then we get

$$
\begin{aligned}
&\left|f(x) \int_{a}^{b} \exp (i \alpha t) d t-\exp (i \alpha x) \int_{a}^{b} f(t) d t\right|\left\{\begin{array}{l}
\left\|f^{\prime}-i \alpha f\right\|_{\infty} \int_{a}^{b}|x-t| d t, \text { if } f^{\prime}-i \alpha f \in L_{\infty}[a, b] \\
\left\|f^{\prime}-i \alpha f\right\|_{p}|g(x)| \int_{a}^{b}|x-t|^{1 / q} d t, \\
\text { if } f^{\prime}-i \alpha f \\
\in L_{p}[a, b] \\
p>1, \\
\frac{1}{p}+\frac{1}{q}=1
\end{array}\right. \\
&\left\|f^{\prime}-i \alpha f\right\|_{1},
\end{aligned}
$$

which, after simple calculation, is equivalent with (23).

The details are omitted.

Corollary 7. With the assumptions of Theorem 9 we have the midpoint inequalities

$$
\begin{aligned}
& \left|\frac{\exp \left(i \alpha\left(\frac{b-a}{2}\right)\right)-\exp \left(-i \alpha\left(\frac{b-a}{2}\right)\right)}{i \alpha} f\left(\frac{a+b}{2}\right)-\int_{a}^{b} f(t) d t\right| \\
& \leq \begin{cases}\frac{1}{4}\left\|f^{\prime}-i \alpha f\right\|_{\infty}(b-a)^{2}, \text { if } f^{\prime}-i \alpha f \in L_{\infty}[a, b], \\
\frac{1}{2^{1 / q}(1+1 / q)}(b-a)^{1+1 / q}\left\|f^{\prime}-i \alpha f\right\|_{p}, & \text { if } f^{\prime}-i \alpha f \in L_{p}[a, b] \\
p>1, \frac{1}{q}=1,\end{cases}
\end{aligned}
$$

or, equivalently 


$$
\begin{aligned}
& \left|\frac{2 \sin \left(\alpha\left(\frac{b-a}{2}\right)\right)}{\alpha} f\left(\frac{a+b}{2}\right)-\int_{a}^{b} f(t) d t\right| \\
& \leq\left\{\begin{array}{ll}
\frac{1}{4}\left\|f^{\prime}-i \alpha f\right\|_{\infty}(b-a)^{2}, \text { if } f^{\prime}-i \alpha f \in L_{\infty}[a, b], \\
\frac{1}{\frac{1}{2^{1 / q}(1+1 / q)}}(b-a)^{1+1 / q}\left\|f^{\prime}-i \alpha f\right\|_{p}, & \text { if } f^{\prime}-i \alpha f \in L_{p}[a, b]
\end{array}\right]
\end{aligned}
$$

\section{An Application for CBS-Inequality}

The following inequality is well known in the literature as the Cauchy-BunyakovskySchwarz inequality, or the CBS-inequality, for short:

$$
\left|\int_{a}^{b} f(t) g(t) d t\right|^{2} \leq \int_{a}^{b}|f(t)|^{2} d t \int_{a}^{b}|g(t)|^{2} d t,
$$

provided that $f, g \in L_{2}[a, b]$.

We have the following result concerning some reverses of the CBS-inequality:

Theorem 10. Let $f, g:[a, b] \rightarrow \mathbb{C}$ be absolutely continuous functions on the interval $[a, b]$ with $g(t) \neq 0$ for all $t \in[a, b]$. Then

$$
\begin{aligned}
& 0 \leq \int_{a}^{b}|g(t)|^{2} d t \int_{a}^{b}|f(t)|^{2} d t-\left|\int_{a}^{b} f(t) g(t) d t\right|^{2}
\end{aligned}
$$

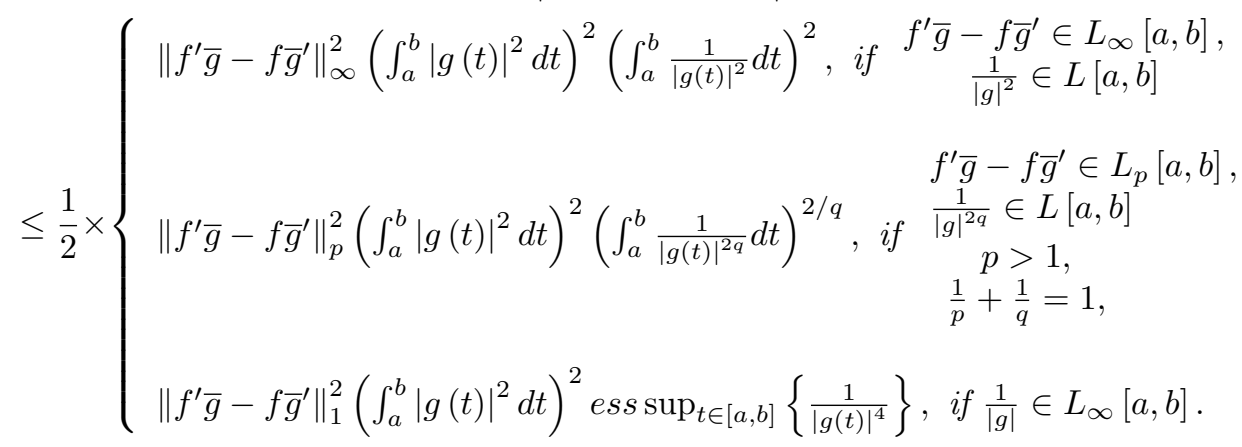


Proof. Utilising the inequality (8) we have

$$
\begin{aligned}
& |\overline{g(t)} f(x)-f(t) \overline{g(x)}| \\
& \leq \begin{cases}\left\|f^{\prime} \bar{g}-f \bar{g}^{\prime}\right\|_{\infty}|g(t) g(x)|\left|\int_{t}^{x} \frac{1}{|g(s)|^{2}} d s\right| & \text { if } f^{\prime} \bar{g}-f \bar{g}^{\prime} \\
& \in L_{\infty}[a, b], \\
\left\|f^{\prime} \bar{g}-f \bar{g}^{\prime}\right\|_{p}|g(t) g(x)|\left|\int_{t}^{x} \frac{1}{|g(s)|^{2 q}} d s\right|^{1 / q} & \text { if } f^{\prime} \bar{g}-f \bar{g}^{\prime} \\
& \in L_{p}[a, b] \\
p>1, \\
\end{cases} \\
& \begin{array}{ll}
p \\
\left\|f^{\prime} \bar{g}-f \bar{g}^{\prime}\right\|_{1}|g(t) g(x)| \sup _{s \in[t, x]([x, t])}\left\{\frac{1}{\left.q(s)\right|^{2}}\right\} .
\end{array}
\end{aligned}
$$

for any $t, x \in[a, b]$.

Taking the square in (28) and integrating over $(t, x) \in[a, b]^{2}$ we have

$$
\begin{aligned}
& \int_{a}^{b} \int_{a}^{b}|\overline{g(t)} f(x)-f(t) \overline{g(x)}|^{2} d t d x \\
& \leq\left\{\begin{array}{l}
\left\|f^{\prime} \bar{g}-f \bar{g}^{\prime}\right\|_{\infty}^{2} \int_{a}^{b} \int_{a}^{b}|g(t) g(x)|^{2}\left|\int_{t}^{x} \frac{1}{|g(s)|^{2}} d s\right|^{2} d t d x, \\
\left\|f^{\prime} \bar{g}-f \bar{g}^{\prime}\right\|_{p}^{2} \int_{a}^{b} \int_{a}^{b}|g(t) g(x)|^{2}\left|\int_{t}^{x} \frac{1}{|g(s)|^{2 q}} d s\right|^{2 / q} d t d x, \\
\left\|f^{\prime} \bar{g}-f \bar{g}^{\prime}\right\|_{1}^{2} \int_{a}^{b} \int_{a}^{b}|g(t) g(x)|^{2} \sup _{s \in[t, x]([x, t])}\left\{\frac{1}{|g(s)|^{4}}\right\} d t d x .
\end{array}\right.
\end{aligned}
$$

Observe that 


$$
\begin{aligned}
& \int_{a}^{b} \int_{a}^{b}|\overline{g(t)} f(x)-f(t) \overline{g(x)}|^{2} d t d x \\
& =\int_{a}^{b} \int_{a}^{b}\left(|g(t)|^{2}|f(x)|^{2}-2 \operatorname{Re}[\overline{g(t)} f(x) \overline{f(t) \overline{g(x)}}]+|g(x)|^{2}|f(t)|^{2}\right) d t d x \\
& =\int_{a}^{b}|g(t)|^{2} d t \int_{a}^{b}|f(x)|^{2} d x-2 R e\left[\int_{a}^{b} \overline{f(t) g(t)} d t \int_{a}^{b} f(x) g(x) d x\right] \\
& +\int_{a}^{b}|g(x)|^{2} d x \int_{a}^{b}|f(t)|^{2} d t \\
& =2\left[\int_{a}^{b}|g(t)|^{2} d t \int_{a}^{b}|f(t)|^{2} d t-\left|\int_{a}^{b} f(t) g(t) d t\right|^{2}\right], \\
& \int_{a}^{b} \int_{a}^{b}\left[|g(t) g(x)|^{2}\left|\int_{t}^{x} \frac{1}{|g(s)|^{2}} d s\right|^{2}\right] d t d x \\
& \leq\left(\int_{a}^{b}|g(t)|^{2} d t\right)^{2}\left(\int_{a}^{b} \frac{1}{|g(t)|^{2}} d t\right)^{2} \\
& \int_{a}^{b} \int_{a}^{b}\left[|g(t) g(x)|^{2}\left|\int_{t}^{x} \frac{1}{|g(s)|^{2 q}} d s\right|^{2 / q}\right] d t d x \\
& \leq\left(\int_{a}^{b}|g(t)|^{2} d t\right)^{2}\left(\int_{a}^{b} \frac{1}{|g(t)|^{2 q}} d t\right)^{2 / q}
\end{aligned}
$$

and

$$
\begin{aligned}
& \int_{a}^{b} \int_{a}^{b}\left[|g(t) g(x)|^{2} \sup _{s \in[t, x]([x, t])}\left\{\frac{1}{|g(s)|^{4}}\right\}\right] d t d x \\
& \leq\left(\int_{a}^{b}|g(t)|^{2} d t\right)^{2} \text { ess } \sup _{t \in[a, b]}\left\{\frac{1}{|g(t)|^{4}}\right\}
\end{aligned}
$$

then by (29) we get the desired result (27).

Acknowledgement. The authors would like to thank the anonymous referee for valuable comments that have been implemented in the final version of the paper. 


\section{References}

[1] A. M. Acu and F. D. Sofonea, On an inequality of Ostrowski type. J. Sci. Arts 2011, no. 3(16), 281-287.

[2] A. M. Acu, A. Baboş and F. D. Sofonea, The mean value theorems and inequalities of Ostrowski type. Sci. Stud. Res. Ser. Math. Inform. 21 (2011), no. 1, 5-16.

[3] S. S. Dragomir, An inequality of Ostrowski type via Pompeiu's mean value theorem. J. Inequal. Pure Appl. Math. 6 (2005), no. 3, Article 83, 9 pp.

[4] A. Ostrowski, Über die Absolutabweichung einer differentienbaren Funktionen von ihren Integralmittelwert, Comment. Math. Hel, 10 (1938), 226-227.

[5] J. Pečarić and Š. Ungar, On an inequality of Ostrowski type, J. Ineq. Pure 85 Appl. Math. 7 (2006). No. 4. Art. 151.

[6] D. Pompeiu, Sur une proposition analogue au théorème des accroissements finis, Mathematica (Cluj, Romania), 22(1946), 143-146.

[7] E. C. Popa, An inequality of Ostrowski type via a mean value theorem, General Mathematics Vol. 15, No. 1, 2007, 93-100.

[8] P. K. Sahoo and T. Riedel, Mean Value Theorems and Functional Equations, World Scientific, Singapore, New Jersey, London, Hong Kong, 2000.

Silvestru Sever DRAGOMIR,

Mathematics, College of Engineering \& Science

Victoria University, PO Box 14428

Melbourne City, MC 8001, Australia.

DST-NRF Center of Excellence in the Mathematical and Statistical Sciences,

School of Computer Science \& Applied Mathematics,

University of the Witwatersrand,

Private Bag 3, Johannesburg 2050, South Africa.

Email: sever.dragomir@vu.edu.au 